\title{
Identification of putative adhesins of Actinobacillus suis and their homologues in other members of the family Pasteurellaceae
}

\author{
Adina R. Bujold(D) and Janet I. Maclnnes*
}

\begin{abstract}
Background: Actinobacillus suis disease has been reported in a wide range of vertebrate species, but is most commonly found in swine. A. suis is a commensal of the tonsils of the soft palate of swine, but in the presence of unknown stimuli it can invade the bloodstream, causing septicaemia and sequelae such as meningitis, arthritis, and death. It is genotypically and phenotypically similar to A. pleuropneumoniae, the causative agent of pleuropneumonia, and to other members of the family Pasteurellaceae that colonise tonsils. At present, very little is known about the genes involved in attachment, colonisation, and invasion by A. suis (or related members of the tonsil microbiota).

Results: Bioinformatic analyses of the A. suis H91-0380 genome were done using BASys and blastx in GenBank. Forty-seven putative adhesin-associated genes predicted to encode 24 putative adhesins were discovered. Among these are 6 autotransporters, 25 fimbriae-associated genes (encoding 3 adhesins), 12 outer membrane proteins, and 4 additional genes (encoding 3 adhesins). With the exception of 2 autotransporter-encoding genes (aidA and $y c g$ V), both with described roles in virulence in other species, all of the putative adhesin-associated genes had homologues in A. pleuropneumoniae. However, the majority of the closest homologues of the A. suis adhesins are found in A. ureae and A. capsulatus - species not known to infect swine, but both of which can cause systemic infections.

Conclusions: A. suis and A. pleuropneumoniae share many of the same putative adhesins, suggesting that the different diseases, tissue tropism, and host range of these pathogens are due to subtle genetic differences, or perhaps differential expression of virulence factors during infection. However, many of the putative adhesins of $A$. suis share even greater homology with those of other pathogens within the family Pasteurellaceae. Similar to A. suis, these pathogens (A. capsulatus and A. ureae) cause systemic infections and it is tempting to speculate that they employ similar strategies to invade the host, but more work is needed before that assertion can be made. This work begins to examine adhesin-associated factors that allow some members of the family Pasteurellaceae to invade the bloodstream while others cause a more localised infection.
\end{abstract}

Keywords: Actinobacillus suis, Pasteurellaceae, Adhesins, Bioinformatics

\section{Background}

Actinobacillus suis, a member of the family Pasteurellaceae, is a Gram negative, facultative anaerobe, and a common commensal of the tonsils of the soft palate of swine [1]. However, under unknown conditions, it can invade the bloodstream of animals of all ages, resulting in septicaemia and sequelae such as meningitis, arthritis,

*Correspondence: macinnes@uoguelph.ca

Department of Pathobiology, Ontario Veterinary College, University

of Guelph, Ontario N1G 2W1, Canada and pneumonia [2]. A. pleuropneumoniae is a primary pathogen of swine that also colonises the upper respiratory tract and causes a contagious pleuropneumonia [3]. A. pleuropneumoniae and A. suis share many of the same virulence factors, including virtually identical ApxI and ApxII toxins (though there are differences in the apxIBD transport genes), iron acquisition proteins including transferrin-binding proteins, urease, lipopolysaccharide, and adhesins [4]. Despite many similarities, 
A. pleuropneumoniae and A. suis cause different diseases in swine, and $A$. suis has a broader host range [5].

Little is known about the virulence factors of A. suis, particularly the adhesins. Therefore, the objective of this study was to use bioinformatics tools to mine the newly annotated genome of a clinical isolate of $A$. suis [6] and identify adhesin-associated genes that may be involved in the early stages of pathogenesis of this organism. Adhesins play an important role in the pathogenesis of most bacteria by allowing them to attach to, colonise, and invade their hosts. In addition to host-pathogen interactions, adhesins are also critical in adherence to abiotic surfaces, auto-aggregation to other bacteria, and in the early stages of biofilm formation [7-9]. Adhesins are often classified as either fimbrial or afimbrial, where fimbrial adhesins are composed of multiple copies of one protein assembled into long appendages such as pili, and afimbrial adhesins are single proteins (e.g., autotransporters or outer membrane proteins) that have adhesive properties [10].

In the current study, we have identified proteins belonging to four different classes of adhesin-associated genes present in the A. suis genome (one fimbrial and three afimbrial) and provided a brief summary of their described roles in attachment in other members of the family Pasteurellaceae, with special emphasis on species in the genus Actinobacillus.

\section{Results and discussion}

Forty-seven putative adhesin-associated genes predicted to encode 24 adhesins were identified in the A. suis H910380 genome. These genes were categorised as autotransporter-encoding (Table 1), fimbriae-associated adhesins (Table 2), outer membrane proteins (OMPs; Table 3), and miscellaneous adhesins (Table 4).

\section{Autotransporters}

Six autotransporter-encoding genes were identified in the A. suis genome (Table 1). Among these, 4 encode proteins that belong to the subfamily known as trimeric autotransporter adhesins (TAAs). Autotransporters are large proteins with three domains-an $\mathrm{N}$-terminal signal domain (present in the immature form of the protein, cleaved from the mature protein), a passenger domain, and a C-terminal translocator domain. In the case of TAAs, the translocator domain is short, and the adhesin structure is formed by a homotrimerisation of the encoded protein [11]. Examples of classic TAAs include Hia in Haemophilus influenzae [12] and YadA in Yersinia enterocolitica $[13,14]$, and they are characterised by a conserved YadA domain and resistance to proteolytic degradation. All TAAs described to date have adhesive properties and bind to different host components including epithelial cells, extracellular matrix components, and circulating molecules (e.g., complement inhibitory proteins, immunoglobulins) [11].

The four genes encoding TAAs identified in the $A$. suis genome, ASU2_04675, ASU2_06645, ASU2_07040, and ASU2_11275, are all well conserved in A. capsulatus ( $\mathrm{E}$ value $=0.0$ ). They also have homologues in $A$. pleuropneumoniae (E values $=0.0-5 \mathrm{e}-25$ ), but the top homologues are found in different serovars. These TAAs also share homology with genes in distant species ( $E$ values ranging from $2 \mathrm{e}-14$ to $2 \mathrm{e}-90$ ). Given that many of the distant species (e.g., Collimonas, Megasphaera, Advenella, Acinetobacter spp.) with homologues of the A. suis-encoded TAAs are environmental isolates, this may hint that these TAAs are well conserved throughout evolution.

The other two autotransporter genes identified in the A. suis genome encode putative conventional autotransporters. These proteins have the same domains as TAAs, but have a longer translocator domain. In addition to being adhesins that play important roles in attachment and biofilm formation, these autotransporters can have additional properties such as cytotoxic, proteolytic or lipolytic activity, and may play a role in serum resistance [11]. In the $A$. suis genome, the putative conventional autotransporter-encoding genes, ASU2_07665 and ASU2_11100, are annotated as $y c g V$ and aidA, respectively. While the $y c g V$ gene is well conserved in $A$. capsulatus $(\mathrm{E}$-value $=0.0$ ) and aidA is quite well conserved in $A$. ureae ( $\mathrm{E}$ value $=5 \mathrm{e}-132$ ), there were no close homologues in A. pleuropneumoniae. It is also noteworthy that in a search for motifs in aidA done using Pfam, no conserved motifs, including the hallmark domains of conventional autotransporters, were detected. Therefore, the classification as an autotransporter-encoding gene relied solely on homology to other autotransporter-encoding genes in GenBank and annotation by BASys. The top homologue of aidA identified in species outside the family Pasteurellaceae was in the Gram positive bacterium Streptococcus suis. However, almost all aidA homologues in Streptococcus species are annotated as hypothetical proteins (with the exception of one homologue which is annotated as the LPXTG-motif cell wall anchor domain protein), and the E value (3e-11), coverage (53\%), and identity (31\%) of the top Streptococcus suis homologue suggest that the degree of conservation of this gene is low. The homology of the A. suis aidA gene with species such as streptococci that share a common environment in the upper respiratory tract of swine may hint at convergent evolution or horizontal gene transfer, but further studies would have to be done to rigorously test such assertions. 
Table 1 Putative autotransporter-encoding genes

\begin{tabular}{|c|c|c|c|c|c|c|c|c|}
\hline \multirow{2}{*}{$\begin{array}{l}\text { ASU2 locus tag } \\
\text { ASU2_04675 }\end{array}$} & \multirow{2}{*}{$\begin{array}{l}\begin{array}{l}\text { Genlnfo (GI) } \\
\text { number }\end{array} \\
407388580\end{array}$} & \multirow{2}{*}{$\begin{array}{l}\text { (Possible) gene } \\
\text { name } \\
-^{\mathrm{a}}\end{array}$} & \multicolumn{3}{|c|}{$\begin{array}{l}\text { GenBank Anno- Top App homologue/E } \\
\text { tated protein value } \\
\text { function }\end{array}$} & \multicolumn{2}{|c|}{$\begin{array}{l}\text { Top Pasteurellaceae } \\
\text { homologue/E value }\end{array}$} & \multirow{2}{*}{$\begin{array}{l}\text { Top other } \\
\text { homologue/E value } \\
\text { Collimonas fungi- } 1 \text { e-54 } \\
\text { vorans Ter } 331\end{array}$} \\
\hline & & & $\begin{array}{l}\text { Autotransporter } \\
\text { adhesin }\end{array}$ & $\begin{array}{r}\text { Ser. } 10 \text { str. } \\
\text { D13039 }\end{array}$ & $2 e-124$ & $\begin{array}{l}\text { Actinobacillus } \\
\text { capsulatus }\end{array}$ & 0.0 & \\
\hline ASU2_06645 & 407388974 & $-^{a}$ & $\begin{array}{l}\text { autotransporter } \\
\text { adhesin }\end{array}$ & Ser. 2 str. 4226 & 0.0 & $\begin{array}{l}\text { Actinobacillus } \\
\text { capsulatus }\end{array}$ & 0.0 & $\begin{array}{l}\text { Megasphaera } \quad 2 \mathrm{e}-90 \\
\text { genomosp. } \\
\text { Type } 1\end{array}$ \\
\hline ASU2_07040 & 407389053 & $-^{a}$ & $\begin{array}{l}\text { extracellular } \\
\text { matrix protein } \\
\text { adhesin A }\end{array}$ & Ser. 13 str. N273 & $5 e-25$ & $\begin{array}{l}\text { Actinobacillus } \\
\text { capsulatus }\end{array}$ & 0.0 & $\begin{array}{l}\text { Advenella mimi- } 2 \mathrm{e}-14 \\
\text { gardefordensis } \\
\text { DPN7 }\end{array}$ \\
\hline ASU2_07665 & 407389178 & $y c g V(t i b A)^{a, b}$ & $\begin{array}{l}\text { outer membrane } \\
\text { autotransporter }\end{array}$ & Ser. 2 str. S1536 & 1.3 & $\begin{array}{l}\text { Actinobacillus } \\
\text { capsulatus }\end{array}$ & 0.0 & $\begin{array}{l}\text { Snodgrassella alvi } 2 \mathrm{e}-37 \\
\text { wkB2 }\end{array}$ \\
\hline ASU2_11100 & 407389854 & $\operatorname{aid} A^{a, b, c}$ & $\begin{array}{l}\text { putative pertacting } \\
\text { family virulence } \\
\text { factor, OM } \\
\text { autotrans- } \\
\text { porter/Type V } \\
\text { secretory path- } \\
\text { way, adhesin }\end{array}$ & Ser. 7 str. AP76 & 1.2 & $\begin{array}{l}\text { Actinobacillus } \\
\text { ureae }\end{array}$ & $5 E-132$ & $\begin{array}{l}\text { Streptococcus suis } 3 e-11 \\
\quad \text { R61 }\end{array}$ \\
\hline ASU2_11275 & 407389889 & $-^{a}$ & $\begin{array}{l}\text { autotransporter } \\
\text { adhesin }\end{array}$ & Ser. 6 str. Femo & $2 e-58$ & $\begin{array}{l}\text { Actinobacillus } \\
\text { capsulatus }\end{array}$ & 0.0 & $\begin{array}{l}\text { Acinetobacter sp. 5e-65 } \\
\text { ANC } 4105\end{array}$ \\
\hline \multicolumn{9}{|c|}{ () indicates a suggested name that was not present in the annotation } \\
\hline \multicolumn{9}{|c|}{ a Function assigned by conserved motifs } \\
\hline \multicolumn{9}{|c|}{ b Identified by BASys } \\
\hline \multicolumn{9}{|c|}{ ' Classified by description of homologues } \\
\hline
\end{tabular}

\section{Fimbriae-associated adhesins}

Twenty-five putative fimbriae-associated adhesin genes were also identified (Table 2). These included 14 genes predicted to be part of a tight adherence (tad) locus, a type IV pilus operon (4 genes), another type IV pilus biogenesis locus containing 6 genes, and another pilusassociated gene.

The pilF gene (ASU2_00450) annotated as a putative fimbrial biogenesis and twitching motility protein, is well conserved in Pasteurellaceae. It is less well conserved outside the family, but pilF homologues are present in Pseudomonas aeruginosa and in Neisseria meningitis (pilW) and are thought to encode a protein that is critical for pilus stability and function, including attachment to human cells $[15,16]$. Pfam analysis revealed TPR repeats in the $A$. suis pilF gene. In other species, these repeats are thought to play a role in protein-protein interactions in both prokaryotic and eukaryotic cells, and contribute to virulence of bacterial pathogens by aiding in attachment to and invasion of host cells and circumventing host defences [17].

The tad locus is a conserved widespread colonisation island [18] that plays an important role in pathogenesis, biofilm formation, and colonisation of several organisms, including members of the family Pasteurellaceae [19-22]. The tad locus encodes the machinery needed to assemble the fimbrial low-molecular-weight protein (Flp) pilin into long, bundled type IVb pili [21, 22]. The A. suis genome contains a tad locus comprised of homologues of flp1-flp2-tadV-rcpC-rcpA-rcpB-tadZ-tadA-tadB-tadCtadD-tadE-tadF-tadG. The two putative pilin genes, ASU2_04295 and ASU2_04300, are predicted to encode $f l p 1$ and $f l p 2$, respectively; however, it may be noted that flp2 is not expressed in Aggregatibacter actinomycetemcomitans [22]. Neither of these genes is very highly conserved within the family Pasteurellaceae and is even less so in more distant species. This may reflect the fact that the $f l p 1$ and $f l p 2$ putative pilin genes in A. suis have adapted for colonisation of different hosts or different host cell receptors. A genetic analysis of the tad locus by Li et al. [23] revealed that $f l p 1$ is truncated or missing altogether in some strains of $A$. pleuropneumoniae. In the same study, these authors found that $t a d C$ is the best conserved among A. pleuropneumoniae strains tested and $\operatorname{tad} G$ the least, findings that were not observed in this work when the same genes in the A. suis genome were compared to other species. However, many of the biogenesis components of the tad locus of A. suis are well conserved in A. pleuropneumoniae and other members of the family Pasteurellaceae such as A. capsulatus, and much less well conserved outside the family.

In addition to the tad locus, the $A$. suis genome also has two other loci for type IV pilus biogenesis: a type IV pilus locus (pilABCD/apfABCD) and a homologue of 
Table 2 Putative fimbriae-associated genes

\begin{tabular}{|c|c|c|c|c|c|c|c|c|c|}
\hline \multirow{2}{*}{$\begin{array}{l}\text { ASU2 locus } \\
\text { tag } \\
\text { ASU2_00450 }\end{array}$} & \multirow{2}{*}{$\begin{array}{l}\begin{array}{l}\text { Genlnfo (GI) } \\
\text { number }\end{array} \\
407387739\end{array}$} & \multirow{2}{*}{$\begin{array}{l}\text { (Possible) } \\
\text { gene name } \\
(\text { pilf })^{c}\end{array}$} & \multirow{2}{*}{ 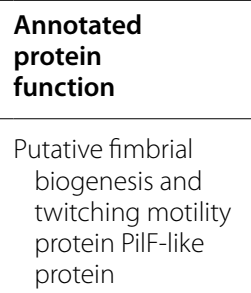 } & \multicolumn{2}{|c|}{$\begin{array}{l}\text { Top App homologue/ } \\
\text { E value }\end{array}$} & \multicolumn{2}{|c|}{$\begin{array}{l}\text { Top Pasteurellaceae } \\
\text { homologue/E value }\end{array}$} & \multicolumn{2}{|c|}{$\begin{array}{l}\text { Top other } \\
\text { homologue/E value }\end{array}$} \\
\hline & & & & Ser. 3 str. JL03 & $2 E-116$ & $\begin{array}{c}\text { Actinobacillus } \\
\text { capsulatus }\end{array}$ & $4 e-114$ & $\begin{array}{l}\text { Vibrio nigripul- } \\
\text { chritudo }\end{array}$ & $3 e-28$ \\
\hline ASU2_04295 & 407388504 & flp1 & flp operon protein & Ser. 4 str. M62 & $1 e-24$ & $\begin{array}{c}\text { Actinobacillus } \\
\text { capsulatus }\end{array}$ & $2 E-27$ & Vibrio owensii & $2 e-05$ \\
\hline ASU2_04300 & 407388505 & $(f l p 2)^{b}$ & $\begin{array}{l}\text { Hypothetical } \\
\text { protein }\end{array}$ & Ser. 7 str. AP76 & $1 e-16$ & $\begin{array}{l}\text { Actinobacillus } \\
\text { ureae }\end{array}$ & $3 E-24$ & $\begin{array}{l}\text { Vibrio sp. } \\
\text { HENC-02 }\end{array}$ & 4.3 \\
\hline ASU2_04305 & 407388506 & $(\operatorname{tad} V)^{c}$ & $\begin{array}{l}\text { flp operon protein B; } \\
\text { Flp pilus assembly } \\
\text { protein, protease } \\
\text { CpaA }\end{array}$ & Ser. 5b str. L20 & $9 E-69$ & $\begin{array}{c}\text { Actinobacillus } \\
\text { capsulatus }\end{array}$ & $6 e-55$ & $\begin{array}{r}\text { Yersinia rohdei } \\
\text { ATCC } 43380\end{array}$ & $5 e-23$ \\
\hline ASU2_04310 & 407388507 & $(\operatorname{rcp} C)$ & flp operon protein $C$ & Ser. 4 str. M62 & $3 e-123$ & $\begin{array}{c}\text { Actinobacillus } \\
\text { capsulatus }\end{array}$ & $3 E-164$ & Yersinia similis & $2 e-19$ \\
\hline ASU2_04315 & 407388508 & $(r c p A)$ & $\begin{array}{l}\text { Rough colony } \\
\text { protein A; flp pilus } \\
\text { assembly protein, } \\
\text { secretin CpaC }\end{array}$ & Ser. 7 str. AP76 & 0.0 & $\begin{array}{l}\text { Actinobacillus } \\
\text { capsulatus }\end{array}$ & 0.0 & Yersinia similis & $1 e-101$ \\
\hline ASU2_04320 & 407388509 & $r c p B$ & $\begin{array}{l}\text { Rough colony } \\
\text { protein B }\end{array}$ & $\begin{array}{l}\text { Ser. } 12 \text { str. } \\
\quad 1096\end{array}$ & $4 e-84$ & $\begin{array}{l}\text { Actinobacillus } \\
\text { capsulatus }\end{array}$ & $1 E-102$ & $\begin{array}{r}\text { Ochotona } \\
\text { princeps }\end{array}$ & 2.3 \\
\hline ASU2_04325 & 407388510 & $(\operatorname{tad} Z)^{c}$ & $\begin{array}{l}\text { flp pilus assembly } \\
\text { protein, ATPase; } \\
\text { CpaE }\end{array}$ & Ser. 5b str. L20 & 0.0 & $\begin{array}{c}\text { Actinobacillus } \\
\text { capsulatus }\end{array}$ & 0.0 & $\begin{array}{l}\text { Yersinia ber- } \\
\text { covieri ATCC } \\
43970\end{array}$ & $3 e-58$ \\
\hline ASU2_04330 & 407388511 & $\operatorname{tad} A$ & $\begin{array}{l}\text { Tight adherence } \\
\text { protein A; Flp pilus } \\
\text { assembly protein, } \\
\text { ATPase CpaF }\end{array}$ & Ser. 5b str. L20 & 0.0 & $\begin{array}{l}\text { Actinobacillus } \\
\text { capsulatus }\end{array}$ & 0.0 & $\begin{array}{l}\text { Yersinia } \\
\text { aldovae ATCC } \\
35236\end{array}$ & $4 e-179$ \\
\hline ASU2_04335 & 407388512 & $\operatorname{tad} B$ & $\begin{array}{l}\text { Tight adherence } \\
\text { protein B; Flp pilus } \\
\text { assembly protein } \\
\text { TadB }\end{array}$ & Ser. 1 str. 4074 & 0.0 & $\begin{array}{l}\text { Actinobacillus } \\
\text { capsulatus }\end{array}$ & 0.0 & $\begin{array}{l}\text { Serratia } \\
\text { marcescens } \\
\text { VGH107 }\end{array}$ & $5 e-64$ \\
\hline ASU2_04340 & 407388513 & $\operatorname{tad} C$ & $\begin{array}{l}\text { Tight adherence } \\
\text { protein C; Flp pilus } \\
\text { assembly protein } \\
\text { TadC }\end{array}$ & $\begin{array}{l}\text { Ser. } 13 \text { str. } \\
\quad \mathrm{N}-273\end{array}$ & $8 e-160$ & $\begin{array}{c}\text { Actinobacillus } \\
\text { capsulatus }\end{array}$ & $6 E-164$ & $\begin{array}{l}\text { Serratia } \\
\text { marcescens } \\
\text { VGH107 }\end{array}$ & $9 e-36$ \\
\hline ASU2_04345 & 407388514 & $\operatorname{tad} D$ & $\begin{array}{l}\text { Tight adherence } \\
\text { protein D; Flp } \\
\text { pilus assembly } \\
\text { protein TadD, con- } \\
\text { tains TPR repeats }\end{array}$ & $\begin{array}{l}\text { Ser. } 13 \text { str. } \\
\quad \mathrm{N}-273\end{array}$ & $3 e-129$ & $\begin{array}{l}\text { Actinobacillus } \\
\text { capsulatus }\end{array}$ & $8 E-148$ & $\begin{array}{l}\text { Hafnia alvei } \\
\text { ATCC } 51873\end{array}$ & $7 e-52$ \\
\hline ASU2_04350 & 407388515 & $\operatorname{tad} E$ & $\begin{array}{l}\text { Tight adherence } \\
\text { protein } \mathrm{E}\end{array}$ & $\begin{array}{l}\text { Ser. } 10 \text { str. } \\
\text { D13039 }\end{array}$ & $2 E-86$ & $\begin{array}{l}\text { Actinobacillus } \\
\text { capsulatus }\end{array}$ & $3 e-84$ & $\begin{array}{l}\text { Serratia } \\
\text { marcescens } \\
\text { VGH107 }\end{array}$ & $2 e-25$ \\
\hline ASU2_04355 & 407388516 & $\operatorname{tad} F$ & $\begin{array}{l}\text { Tight adherence } \\
\text { protein } \mathrm{F}\end{array}$ & Ser. 6 str. Femo & $2 E-66$ & $\begin{array}{l}\text { Actinobacillus } \\
\text { capsulatus }\end{array}$ & $1 e-59$ & $\begin{array}{l}\text { Yersinia entero- } \\
\text { colitica }\end{array}$ & $2 e-12$ \\
\hline ASU2_04360 & 407388517 & $\operatorname{tad} G$ & $\begin{array}{l}\text { Tight adherence } \\
\text { protein } \mathrm{G}\end{array}$ & $\begin{array}{l}\text { Ser. } 2 \text { str. } \\
\text { S1536 }\end{array}$ & 0.0 & $\begin{array}{l}\text { Mannheimia } \\
\text { haemolytica }\end{array}$ & 0.0 & $\begin{array}{l}\text { Yersinia fred- } \\
\text { eriksenii ATCC } \\
33641\end{array}$ & $6 e-19$ \\
\hline ASU2_05030 & 407388651 & $(p i l D)^{c}$ & $\begin{array}{l}\text { Fimbrial leader } \\
\text { peptidase; Type } \\
\text { II secretory } \\
\text { pathway, prepilin } \\
\text { signal peptidase } \\
\text { PulO and related } \\
\text { peptidases }\end{array}$ & Ser. 1 str. 4074 & $3 e-56$ & $\begin{array}{l}\text { Actinobacillus } \\
\text { ureae }\end{array}$ & $1 E-135$ & $\begin{array}{l}\text { Enterococcus } \\
\text { faecium } \\
\text { EnGen0131 }\end{array}$ & 0.014 \\
\hline
\end{tabular}


Table 2 continued

\begin{tabular}{|c|c|c|c|c|c|c|c|c|c|}
\hline \multirow{2}{*}{$\begin{array}{l}\text { ASU2 locus } \\
\text { tag } \\
\text { ASU2_05035 }\end{array}$} & \multirow{2}{*}{$\begin{array}{l}\begin{array}{l}\text { Genlnfo (GI) } \\
\text { number }\end{array} \\
407388652 \\
\end{array}$} & \multirow{2}{*}{$\begin{array}{l}\text { (Possible) } \\
\text { gene name } \\
(p i / C)^{c}\end{array}$} & \multirow{2}{*}{$\begin{array}{l}\begin{array}{l}\text { Annotated } \\
\text { protein } \\
\text { function }\end{array} \\
\text { Pili/fimbriae biogen- } \\
\text { esis protein; Type } \\
\text { II secretory path- } \\
\text { way, component } \\
\text { PulF }\end{array}$} & \multicolumn{2}{|c|}{$\begin{array}{l}\text { Top App homologue/ } \\
\text { E value }\end{array}$} & \multicolumn{2}{|c|}{$\begin{array}{l}\text { Top Pasteurellaceae } \\
\text { homologue/E value }\end{array}$} & \multicolumn{2}{|c|}{$\begin{array}{l}\text { Top other } \\
\text { homologue/E value }\end{array}$} \\
\hline & & & & Ser. 7 str. AP76 & $5 e-112$ & $\begin{array}{l}\text { Actinobacillus } \\
\text { ureae }\end{array}$ & 0.0 & $\begin{array}{l}\text { Glaciecola } \\
\text { punicea DSM } \\
14233\end{array}$ & $2 e-29$ \\
\hline ASU2_05040 & 407388653 & $(h \circ f B, p i l B)^{c}$ & $\begin{array}{l}\text { Fimbrial biogenesis } \\
\text { protein; Type II } \\
\text { secretory path- } \\
\text { way, ATPase PulE/ } \\
\text { Tfp pilus assembly } \\
\text { pathway, ATPase } \\
\text { PilB }\end{array}$ & Ser. 5b str. L20 & 0.0 & $\begin{array}{l}\text { Actinobacillus } \\
\text { ureae }\end{array}$ & 0.0 & $\begin{array}{l}\text { Plesiomonas } \\
\text { shigelloides } \\
302-73\end{array}$ & $3 e-117$ \\
\hline ASU2_05045 & 407388654 & $\begin{array}{l}a p f A(p p d D \\
\text { pilA })^{c}\end{array}$ & $\begin{array}{l}\text { Type } 4 \text { prepilin } \\
\text { subunit; Tfp pilus } \\
\text { assembly protein, } \\
\text { major pilin PilA }\end{array}$ & $\begin{array}{l}\text { Ser. } 12 \text { str. } \\
1096\end{array}$ & $5 e-37$ & $\begin{array}{l}\text { Actinobacillus } \\
\text { ureae }\end{array}$ & $1 E-70$ & $\begin{array}{l}\text { Bacillus sp. } \\
\text { BVB01 }\end{array}$ & $5 e-20$ \\
\hline ASU2_06115 & 407388868 & hofQ $(c o m E)^{\mathrm{b}, \mathrm{c}}$ & $\begin{array}{l}\text { Type II secretory } \\
\text { pathway, compo- } \\
\text { nent HofQ }\end{array}$ & Ser. 5b str. L20 & 0.0 & $\begin{array}{l}\text { Actinobacillus } \\
\text { ureae }\end{array}$ & 0.0 & $\begin{array}{l}\text { Plesiomonas } \\
\text { shigelloides } \\
302-73\end{array}$ & $3 e-100$ \\
\hline ASU2_06120 & 407388869 & $(c o m D)^{b, c}$ & $\begin{array}{l}\text { Hypothetical pro- } \\
\text { tein; Ribosomal } \\
\text { protein S15P/S13E }\end{array}$ & Ser. 5b str. L20 & $3 e-59$ & $\begin{array}{l}\text { Actinobacillus } \\
\text { ureae }\end{array}$ & $8.00 E-60$ & $\begin{array}{r}\text { Streptomyces } \\
\text { sp. AA0539 }\end{array}$ & 0.049 \\
\hline ASU2_06125 & 407388870 & $(\operatorname{com} C)^{b, c}$ & $\begin{array}{l}\text { Hypothetical } \\
\text { protein }\end{array}$ & Ser. 7 str. AP76 & $9 e-51$ & $\begin{array}{l}\text { Actinobacillus } \\
\text { capsulatus }\end{array}$ & $4.00 E-77$ & $\begin{array}{l}\text { Klebsiella } \\
\text { pneumoniae } \\
\text { MGH } 52\end{array}$ & 0.12 \\
\hline ASU2_06130 & 407388871 & $(c o m B)^{b, c}$ & $\begin{array}{l}\text { Hypothetical } \\
\text { protein }\end{array}$ & Ser. 3 str. JL03 & $2 e-65$ & $\begin{array}{l}\text { Actinobacillus } \\
\text { capsulatus }\end{array}$ & $5 e-95$ & $\begin{array}{l}\text { Tepidiphilus } \\
\text { margaritifer }\end{array}$ & 0.49 \\
\hline ASU2_06135 & 407388872 & $(\operatorname{com} A)^{\mathrm{b}, \mathrm{c}}$ & $\begin{array}{l}\text { Hypothetical } \\
\text { protein }\end{array}$ & $\begin{array}{r}\text { Ser. } 10 \text { str. } \\
\text { D13039 }\end{array}$ & $7 e-106$ & $\begin{array}{l}\text { Actinobacillus } \\
\text { capsulatus }\end{array}$ & $4 E-131$ & $\begin{array}{l}\text { Desulfosarcina } \\
\text { sp. BuS5 }\end{array}$ & 1.9 \\
\hline ASU2_11115 & 407389857 & $\mathrm{comF}^{\mathrm{c}}$ & Competence & $\begin{array}{r}\text { Ser. } 10 \text { str. } \\
\text { D13039 }\end{array}$ & $9 e-97$ & $\begin{array}{l}\text { Actinobacillus } \\
\text { capsulatus }\end{array}$ & $7 E-150$ & $\begin{array}{l}\text { Serratia } \\
\text { fonticola AU- } \\
\text { AP2C }\end{array}$ & $7 e-47$ \\
\hline
\end{tabular}

() indicates a suggested name that was not present in the annotation

a Function assigned by conserved motifs

b Identified by BASys

c Classified by description of homologues

the com $A B C D E F$ locus. Type IV pili are important virulence factors in many Gram negative organisms, including other members of the family Pasteurellaceae such as nontypeable Haemophilus influenzae (NTHi) [24-27], Pasteurella multocida [28], and A. pleuropneumoniae [29-31]. In these species, type IV pili have demonstrated roles in biofilm formation, attachment to epithelial cells, twitching motility, competence, and interactions with phage [32-34]. In A. pleuropneumoniae, the apfA pilin gene is present in all strains and is well conserved in all serovars [31]. The homologue of this gene in $A$. suis (ASU2_05045) is the least well conserved gene in the pilABCD locus, but is still homologous to genes in both $A$. ureae (1e-70) and A. pleuropneumoniae (5e-37). Of the biogenesis genes, pilBCD, pilB, which encodes the ATPase, is the best conserved ( $\mathrm{E}$ values $=0.0$ ), and has well conserved homologues outside the family Pasteurellaceae (e.g., in Plesiomonas shigelloides, E value $=5 \mathrm{e}-20)$. On the other hand, the pilD gene, predicted to encode the fimbrial leader peptidase, is not conserved in species outside the family Pasteurellaceae (e.g., Enterococcus faecium, E value $=0.014$ ).

Like pilABCD/apfABCD, the comABCDEF competence locus is predicted to encode the biogenesis components for type IV pilus assembly; however, no pilin gene is associated with this operon in the A. suis genome, and the comF gene (ASU2_11115) is not linked with the rest of the com locus, unlike other species such as NTHi [24]. In a recent study of NTHi, Carruthers et al. found that all of the products of both the pil and com operons, including $\operatorname{com} F$, are essential for proper type IV pilus construction and formation [24]. Taken together, these results suggest that the proteins encoded by the pil and com loci may work together to produce type IV pili in A. suis, and that the pilA homologue (ASU2_05045) may encode the major pilin protein. 
Table 3 Putative outer membrane protein genes

\begin{tabular}{|c|c|c|c|c|c|c|c|c|c|}
\hline \multirow{2}{*}{$\begin{array}{l}\text { ASU2 locus } \\
\text { tag } \\
\text { ASU2_00030 }\end{array}$} & \multirow{2}{*}{$\begin{array}{l}\begin{array}{l}\text { Genlnfo (GI) } \\
\text { number }\end{array} \\
407387657 \\
\end{array}$} & \multirow{2}{*}{$\begin{array}{l}\text { (Possible) } \\
\text { gene name } \\
\text { ompP2 }\end{array}$} & \multirow{2}{*}{$\begin{array}{l}\text { Annotated } \\
\text { protein } \\
\text { function }\end{array}$} & \multicolumn{2}{|c|}{$\begin{array}{l}\text { Top App homologue/ } \\
\text { E value }\end{array}$} & \multicolumn{2}{|c|}{$\begin{array}{l}\text { Top Pasteurellaceae } \\
\text { homologue/E value }\end{array}$} & \multicolumn{2}{|c|}{$\begin{array}{l}\text { Top other homologue/ } \\
\text { E value }\end{array}$} \\
\hline & & & & Ser. 5b str. L20 & 0.0 & $\begin{array}{l}\text { Actinobacillus } \\
\text { capsulatus }\end{array}$ & 0.0 & $\begin{array}{l}\text { Neisseria sp. oral } \\
\text { taxon } 014 \text { str. } \\
\text { F0314 }\end{array}$ & $6 e-131$ \\
\hline ASU2_00525 & 407387754 & ompP2 & $\begin{array}{l}\text { Outer mem- } \\
\text { brane protein } \\
\text { P2; porin }\end{array}$ & Ser. 4 str. M62 & $6 e-42$ & $\begin{array}{l}\text { Actinobacillus } \\
\text { ureae }\end{array}$ & $9 E-159$ & $\begin{array}{l}\text { Neisseria sp. oral } \\
\text { taxon } 014 \text { str. } \\
\text { F0314 }\end{array}$ & $4 e-08$ \\
\hline ASU2_01965 & 407388042 & $(o m p P 1)^{c}$ & $\begin{array}{l}\text { Long-chain } \\
\text { fatty acid } \\
\text { outer } \\
\text { membrane } \\
\text { transporter }\end{array}$ & Ser. 7 str. AP76 & $9 e-142$ & $\begin{array}{l}\text { Actinobacillus } \\
\text { ureae }\end{array}$ & 0.0 & $\begin{array}{l}\text { Serratia protea- } \\
\text { maculans } 568\end{array}$ & $4 e-98$ \\
\hline ASU2_02415 & 407388132 & $-b, c$ & $\begin{array}{l}\text { Hypothetical } \\
\text { protein }\end{array}$ & Ser. 5b str. L20 & $3 e-122$ & $\begin{array}{l}\text { Actinobacillus } \\
\text { ureae }\end{array}$ & $2 E-132$ & Pantoea sp. A4 & $6 e-06$ \\
\hline ASU2_03005 & 407388248 & plp4 & $\begin{array}{l}\text { Lipoprotein; } \\
\text { small protein } \\
\text { A (tmRNA- } \\
\text { binding) }\end{array}$ & Ser. 5b str. L20 & 0.0 & $\begin{array}{l}\text { Actinobacillus } \\
\text { capsulatus }\end{array}$ & 0.0 & $\begin{array}{l}\text { Pelistega sp. } \\
\text { HM-7 }\end{array}$ & $8 e-107$ \\
\hline ASU2_03810 & 407388407 & - & $\begin{array}{l}\text { Outer mem- } \\
\text { brane protein } \\
\text { P2-like pro- } \\
\text { tein; porin }\end{array}$ & Ser. 3 str. JL03 & 0.0 & $\begin{array}{l}\text { Actinobacillus } \\
\text { ureae }\end{array}$ & 0.0 & $\begin{array}{l}\text { Neisseria sp. oral } \\
\text { taxon } 014\end{array}$ & $7 e-27$ \\
\hline ASU2_05520 & 407388749 & palA & $\begin{array}{l}\text { Outer mem- } \\
\text { brane protein } \\
\text { and related } \\
\text { peptidogly- } \\
\text { can-associ- } \\
\text { ated (lipo) } \\
\text { proteins }\end{array}$ & Ser. 3 str. JL03 & $2 E-89$ & $\begin{array}{l}\text { Actinobacillus } \\
\text { ureae }\end{array}$ & $2 E-89$ & $\begin{array}{l}\text { Morganella } \\
\text { morganii } \\
\text { subsp. morga- } \\
\text { nii KT }\end{array}$ & $1 e-46$ \\
\hline ASU2_05735 & 407388792 & ompW & $\begin{array}{l}\text { Outer mem- } \\
\text { brane protein }\end{array}$ & Ser. 5b str. L20 & $2 e-68$ & $\begin{array}{l}\text { Actinobacillus } \\
\text { capsulatus }\end{array}$ & $2 E-119$ & Vibrio sp. Ex25 & $4 e-42$ \\
\hline ASU2_06455 & 407388936 & $-{ }^{b, c}$ & $\begin{array}{l}\text { Hypothetical } \\
\text { protein }\end{array}$ & Ser. 7 str. AP76 & $2 e-136$ & $\begin{array}{l}\text { Actinobacillus } \\
\text { ureae }\end{array}$ & $2 E-141$ & $\begin{array}{l}\text { Taylorella equig- } \\
\text { enitalis ATCC } \\
35865\end{array}$ & $2 e-54$ \\
\hline ASU2_09935 & 407389622 & ompP5 & $\begin{array}{l}\text { Outer mem- } \\
\text { brane protein } \\
\text { P5 }\end{array}$ & Ser. 3 str. JL03 & 0.0 & $\begin{array}{l}\text { Actinobacillus } \\
\text { capsulatus }\end{array}$ & 0.0 & $\begin{array}{l}\text { Shimwellia } \\
\text { blattae DSM } \\
4481\end{array}$ & $3 e-69$ \\
\hline ASU2_09940 & 407389623 & $(o m p A)^{c}$ & $\begin{array}{l}\text { Major outer } \\
\text { membrane } \\
\text { protein }\end{array}$ & Ser. 6 str. Femo & 0.0 & $\begin{array}{l}\text { Actinobacillus } \\
\text { capsulatus }\end{array}$ & 0.0 & $\begin{array}{l}\text { Salmonella } \\
\text { enterica subsp. } \\
\text { enterica ser. } \\
\text { Typhimurium }\end{array}$ & $2 e-66$ \\
\hline ASU2_11270 & 407389888 & plp4 & $\begin{array}{l}\text { Outer mem- } \\
\text { brane protein } \\
\text { and related } \\
\text { peptidogly- } \\
\text { can-associ- } \\
\text { ation (lipo) } \\
\text { proteins }\end{array}$ & Ser. 4 str. M62 & $3 e-35$ & $\begin{array}{l}\text { Actinobacillus } \\
\text { ureae }\end{array}$ & $6 E-85$ & $\begin{array}{l}\text { Methylovorus } \\
\text { glucosetro- } \\
\text { phus SIP3-4 }\end{array}$ & $4 e-25$ \\
\hline
\end{tabular}

() indicates a suggested name that was not present in the annotation

a Function assigned by conserved motifs

b Identified by BASys

c Classified by description of homologues

\section{Outer membrane proteins}

Genes predicted to encode twelve outer membrane proteins (OMPs) were identified, including homologues of ompA, ompP2, and ompP5 porin genes (Table 3). OMPs are described as multifunctional proteins. Many OMPs have been demonstrated to form porins in the outer membrane of Gram negative bacteria, which can contribute to nutrient acquisition, antibiotic resistance, 


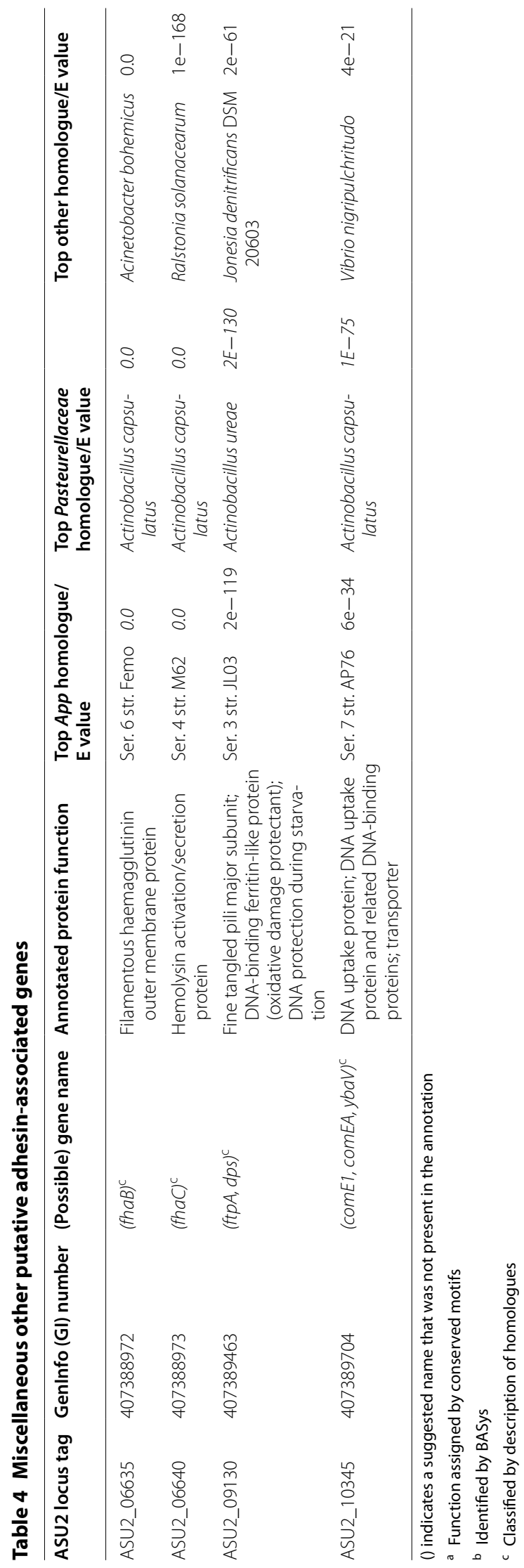


attachment, invasion, and complement resistance, to name a few [35].

Most of the OMPs of A. suis are highly conserved when compared to other members of the family Pasteurellaceae. Two members of the OmpA family were identified in the A. suis genome, ASU2_09940 and ASU2_09935. In our previous studies, the OmpA homologue ASU2_09940 was identified by signature-tagged mutagenesis as an important virulence factor of A. suis, with a demonstrated role in attachment to swine tonsil explants and to porcine brain microvascular endothelial cells $[36,37]$. The other member of the OmpA family of OMPs, an ompP5 homologue (ASU2_09935), is adjacent to the ompA homologue ASU2_09940 in the $A$. suis genome. It is also highly conserved $(\mathrm{E}$ value $=0.0$ ) in members of the family Pasteurellaceae and has a high degree of homology with OMPs outside the family. In NTHi, OmpP5 has been shown to bind to human mucin [38] and to CEACAM1 [39]; however, the precise role of OmpP5 and most other A. suis OMPs in pathogenesis remains to be demonstrated.

Two ompP2 genes (ASU2_00030 and ASU2_00525) and one ompP2-like gene (ASU2_03810) were identified in the $A$. suis genome. In addition to conferring antibiotic resistance [40], providing a pore for general diffusion and transport of specific substrates [41], the OmpP2 of NTHi has also been shown to play a role in attachment in the host environment through interactions with mucin [42]. The ompP2 gene (ASU2_00030) is predicted to encode a protein that is very similar to an OmpP2 homologue in $A$. capsulatus $(\mathrm{E}=0.0)$ while the ASU2_00525 gene encodes a protein that is well conserved in $A$. ureae (9E-159). The ompP2 homologues identified in A. suis are well conserved in A. pleuropneumoniae, but the serovar of the top homologues in A. pleuropneumoniae is different with each gene, as is the degree of conservation. Of the OMPs identified in A. suis, the ompP2 gene ASU2_00525 and the plp4 homologue ASU2_02415 have the least homology with proteins encoded by organisms outside of the family Pasteurellaceae. The GC content of ASU2_00525 differs markedly from that of the A. suis genome (36 vs. $40.24 \%$ ), which may suggest that this ompP2 gene was recently acquired by $A$. suis.

Because of the multifunctional nature of the OMPs, it would be premature to predict that all OMPs identified in this study play a role in attachment or invasion, and further studies should be done to characterise each gene and its potential role in bacterial pathogenesis for $A$. suis.

\section{Miscellaneous adhesins}

Four additional genes from three different loci were identified that could play a role in bacterial attachment, colonisation, or invasion for A. suis (Table 4).
A filamentous haemagglutinin (FHA) locus consisting of two genes (ASU2_06635 and ASU2_06640) is also found in the $A$. suis genome. The fhaB gene encodes the adhesin structure while $f h a C$ encodes the transporter. FHA has been demonstrated to play a role in bacterial attachment to integrins, carbohydrates present on macrophages, cilia, epithelial cells, and extracellular matrix components including heparin [43], and is thought to contribute to colonisation and biofilm formation by important pathogens such as Histophilus somni, Bordetella bronchiseptica, Acinetobacter baumannii, and Pasteurella multocida [44-47]. The A. suis fhaB gene has highly conserved (E values $=0.0$ ) homologues in A. pleuropneumoniae, Pasteurellaceae, and in other species outside of the family Pasteurellaceae. The fhaC gene is also predicted to encode highly conserved homologues in members of the family Pasteurellaceae but to a slightly lower degree ( $\mathrm{E}$ value $=1 \mathrm{e}-168)$. It is also interesting to note that the TAA-encoding ASU2_06645 gene is linked to the filamentous haemagglutinin locus, though the relevance of this finding, if any, remains to be elucidated.

A fine-tangled pili gene, $f t p A$, is also present in the $A$. suis genome. This gene lacks a cleavable signal sequence [48], and no biogenesis genes for the translocation and assembly of this structure were identified. In other species, fine-tangled pili are assigned to the DNA protection during starvation (DPS) family of proteins. DPS proteins are thought to confer protection of DNA from environmental stressors such as low $\mathrm{pH}, \mathrm{Fe}^{2+}$, and hydrogen peroxide [49]. Further, these proteins have been shown to be involved in bacterial adhesion to and invasion of host cells, and in auto-aggregation [49-53], though it is not clear whether the mechanisms of these actions are via a direct or indirect adhesive function of the Dps homologue. In A. suis, the ftpA gene (ASU2_09130) is well conserved in both $A$. pleuropneumoniae ( $\mathrm{E}$ value $=2 \mathrm{e}-119$ ) and other members of Pasteurellaceae (A. ureae, E value $=2 \mathrm{e}-130$ ), and to a lesser extent in other species (Jonesia denitrificans, $\mathrm{E}$ value $=2 \mathrm{e}-61$ ).

Finally, a homologue of comE1, originally described in Pasteurella multocida [54], was also identified in A. suis. In addition to its roles in DNA-binding and uptake, this gene encodes a protein involved in bacterial attachment of five different members of the family Pasteurellaceae to the extracellular matrix component fibronectin $[55,56]$. The closest homologue of the comE1 gene in A. suis (ASU2_10345) is found in A. capsulatus (E value $=1 \mathrm{e}-75$ ). Less well conserved homologues are also present in A. pleuropneumoniae (E value $=6 \mathrm{e}-34$ ), other members of Pasteurellaceae, and even in other species outside the family. Given the role of this gene in fibronectin-binding in other members of Pasteurellaceae, 
it would be interesting to assess whether it plays a similar function in A. suis.

\section{Adhesins in other $A$. suis strains}

To determine whether putative adhesin genes are conserved in other $A$. suis isolates, real-time PCR was done on 9 additional isolates, including A. pleuropneumoniae $\mathrm{L} 20$, a serovar $5 \mathrm{~b}$ isolate (Table 5 ). Ten genes were chosen for characterisation, with representatives from each of the classes of adhesins described. All A. suis isolates tested were positive for the selected adhesin genes, while the A. pleuropneumoniae isolate was only positive for the putative ompP2 gene. Upon closer inspection of the A. pleuropneumoniae L20 genome sequence, the only adhesin gene tested without a homologue was $y \mathrm{cgV}$ (ASU2_07665); however, despite there being homologues of the other genes, the sequence conservation in the primer binding sites in all but the ompP2 gene was poor.

The pseudogenomes of three additional $A$. suis genomes-ATCC 15557, H89-0406, and H91-1173were annotated using BASys, and the genome sequence of ATCC $33416^{\mathrm{T}}$ was obtained from GenBank [57]. These four genome sequences were used to determine whether putative adhesin genes were conserved in different $A$. suis isolates using blastn for direct nucleotide sequence comparisons (Additional file 1). Homologues of all adhesin genes identified in the $A$. suis H91-0380 genome were found in the four additional genomes, and were for the most part highly conserved (most $>99 \%$ sequence identity). Some gene lengths varied among isolates, with the most notable differences seen in the ASU2_04675 autotransporter-encoding homologue found in ATCC 33415 and ATCC 15557, the ASU2_11275 autotransporter-encoding homologue in ATCC 15557, and the truncated but highly conserved flp1 (ASU2_04295) homologue in H89-1173. The OMP homologue ASU2_01965 in the ATCC 33415 isolate shared only $67 \%$ nucleotide identity with H91-0380, despite $90 \%$ sequence coverage. Overall, however, putative adhesin genes were highly conserved in all A. suis isolates examined, which may suggest a clonal population, though other classes of genes, particularly virulence-associated genes, should also be compared.

\section{Conclusions}

Attachment and colonisation of the host environment are important steps in the early stages of bacterial colonisation and pathogenesis [7]. As virtually nothing was known about these early steps in A. suis, the purpose of this study was to identify putative adhesins that may contribute to these processes in the genomes of several $A$. suis strains. Our analysis revealed that $A$. suis shares many of the same putative adhesins as $A$. pleuropneumoniae, an important primary pathogen of swine that is also known to colonise the upper respiratory tract. It may therefore be hypothesised that the different tissue tropisms and diseases caused by $A$. suis and A. pleuropneumoniae might be attributed, at least in part, to subtle differences in the adhesins of these organisms or to differential expression of adhesins at different stages of the infection process.

The adhesins identified in the $A$. suis genome are also well conserved in several other members of the family Pasteurellaceae. It is perhaps noteworthy that Pasteurellaceae that cause similar diseases but in different hosts, such as $A$. ureae and A. capsulatus, have nearly all the same adhesins as are present in $A$. suis. Of particular note are the autotransporter-encoding genes $y c g V$ and aid $A$ that are present in A. suis, A. ureae, and A. capsulatus, but which are missing in $A$. pleuropneumoniae. It may be hypothesised that these organisms employ similar strategies to invade the host, but more work is needed to characterise such host-pathogen interactions.

Together, these data begin to identify attachment and colonisation factors that may allow some members of the family Pasteurellaceae to invade the bloodstream and others to cause more localised infections. Future research on the expression of adhesins in A. suis and other organisms will help in elucidating the mechanisms of attachment and colonisation, and should eventually lead to a better understanding of critical host-pathogen relationships.

\section{Methods}

\section{Bioinformatics}

To identify putative adhesin-associated genes in Actinobacillus suis H91-0380, a virulent O2:K2 isolate [6], a manual search of the annotations of the A. suis H91-0380 genome assigned by the BASys pipeline [58] and GenBank (http://www.ncbi.nlm.nih.gov/) was done to identify putative adhesin-associated genes; blastx was used to find homologues in other species with a described or annotated role in attachment, colonisation, or invasion. Genes or proteins described in the literature in other members of the family Pasteurellaceae were also analysed by blastx or blastp to find homologues in A. suis.

Further analysis of selected putative adhesin-associated genes was done using Pfam (http://pfam.xfam.org/) to determine if conserved amino acid motifs characteristic of described protein families were present. When motifs were not identified, sequence identity and query coverage alone were used to classify genes.

\section{Bacterial strains and growth media}

Bacterial isolates (Table 6) were cultured from glycerol stocks onto Columbia agar plates containing $5 \%$ 


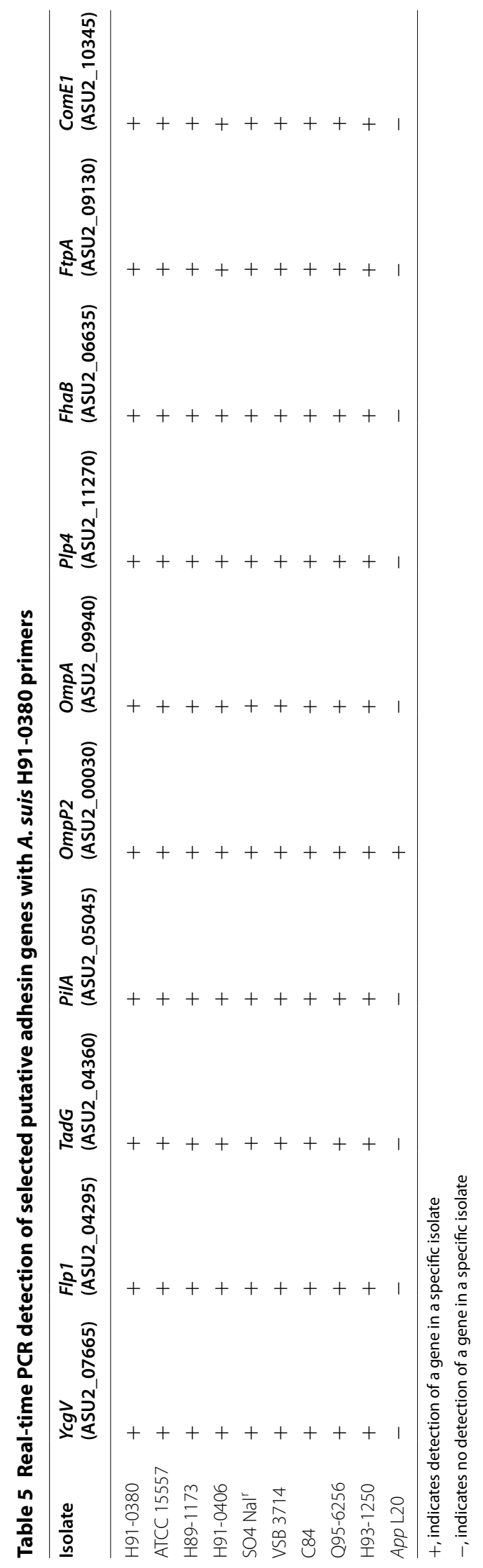


Table 6 Strains used in this study

\begin{tabular}{lll}
\hline Bacterial strain & Characteristic(s) & GenBank accession number and Reference \\
\hline Actinobacillus suis H91-0380 & O2:K2 clinical isolate & CP003875; [6, 61] \\
Actinobacillus suis ATCC 33415 & Untyped clinical isolate & CP009159; [57] \\
Actinobacillus suis ATCC 15557 & O1:K1 isolate & {$[61,62]$} \\
Actinobacillus suis H89-1173 & O2:K3 clinical isolate & {$[61,62]$} \\
Actinobacillus suis H91-0406 & O2:K3 clinical isolate & {$[61,62]$} \\
Actinobacillus suis SO4 Nal & O1:K1 isolate & {$[61,62]$} \\
Actinobacillus suis VSB 3714 & Rough:K? isolate & {$[61,62]$} \\
Actinobacillus suis C84 & O1:K2 isolate & {$[61,62]$} \\
Actinobacillus suis Q95-6256 & Untypable isolate & {$[61,62]$} \\
Actinobacillus suis H93-1250 & Untyped clinical isolate & {$[61,62]$} \\
Actinobacillus pleuropneumoniae L20 & Serovar 5b & {$[63]$}
\end{tabular}

sheep's blood (Oxoid Co., Nepean, ON, USA), and in the case of the A. pleuropneumoniae isolate, supplemented with $0.01 \%(\mathrm{wt} / \mathrm{vol})$ nicotinamide adenine dinucleotide (Sigma-Aldrich, St. Louis, MO). Plates were incubated overnight at $37^{\circ} \mathrm{C}$ in an atmosphere of $5 \% \mathrm{CO}_{2}$.

\section{Real-time PCR}

Crude genomic DNA was prepared by picking isolated colonies and dispersing them in Instagene matrix (BioRad Laboratories Ltd., Hercules, CA), mixing by vortex, incubating at $56{ }^{\circ} \mathrm{C}$ for $30 \mathrm{~min}$, mixing again by vortex, incubating at $100{ }^{\circ} \mathrm{C}$ for $8 \mathrm{~min}$, centrifuging at $5000 \times g$ for $2 \mathrm{~min}$, and using the supernatant as template for PCR. At least two biological replicates were done for each strain and gene tested.

PCR primers were designed using Primer3 as previously described [59], and are listed in Table 7. The total reaction volume was $20 \mu \mathrm{L}$, which contained $10 \mu \mathrm{L}$ LightCycler 480 SYBR Green I Master mix (Roche Diagnostics Co., Indianapolis, IN, USA), $0.4 \mu \mathrm{L}$ each of the forward and reverse primers to a final concentration of $1 \mu \mathrm{M}$, $4.2 \mu \mathrm{L}$ nuclease-free water, and $5 \mu \mathrm{L}$ template.

Table 7 Primers used in this work

\begin{tabular}{|c|c|c|c|c|}
\hline Primer name & Class & Locus tag & Sequence & Source \\
\hline ASU2-ycgV-F1 & Autotransporter & ASU2_07665 & CTGGGATGTTCCTGTTGTTGCT & This work \\
\hline ASU2-ycgV-R1 & & & TTTACCGAGGTTTATCGTACTGTTTGT & This work \\
\hline ASU2-flp1-F1 & Fimbriae-associated & ASU2_04295 & CTGTAACTGAAGGTATCCGCAACT & This work \\
\hline ASU2-flp1-R1 & & & TGCTAAAGCCACAGCAATTAAACC & This work \\
\hline ASU2-tadG-F1 & Fimbriae-associated & ASU2_04360 & ACTGAATGACGACAAGAATACATCG & This work \\
\hline ASU2-tadG-R1 & & & GCAGAGTAGTAGTTTCCATCACCT & This work \\
\hline ASU2-pilA-F1 & Fimbriae-associated & ASU2_05045 & ACTGTTAGCGGCATCTTCTGC & This work \\
\hline ASU2-pilA-R1 & & & CTACGCTGCCCTTGCCATTC & This work \\
\hline ASU2-ompP2-F1 & OMP & ASU2_00030 & ACCTCAGCCAAAGACACTTACCAAA & This work \\
\hline ASU2-ompP2-R1 & & & TAAACGCCATTCTACACGGCCTAAA & This work \\
\hline ASU2-ompA-F1 & OMP & ASU2_09940 & CGGTAAAGTAGGTGTTGCAGTT & This work \\
\hline ASU2-ompA-R1 & & & ATTTCTCTGTTGGTTCGTTAGTGT & This work \\
\hline ASU2-plp4-F1 & OMP & ASU2_11270 & GTCGAATCTAACTGCGAAGGGTAAAG & This work \\
\hline ASU2-plp4-R1 & & & GTTGTATGCAGGAGAACCTAAACGG & This work \\
\hline ASU2-fhaB-F1 & Miscellaneous & ASU2_06635 & GGATTTAGCCGTACATGGAAATGG & This work \\
\hline ASU2-fhaB-R1 & & & ATACTTTACCTTTGATTTGAGCCGT & This work \\
\hline ASU2-ftpA-F1 & Miscellaneous & ASU2_09130 & CGGAGCGTATGGCAGCATTAG & This work \\
\hline ASU2-ftpA-R1 & & & GGATATTCAGGCGTTTGACGTGTT & This work \\
\hline ASU2-comE1-F1 & Miscellaneous & ASU2_10345 & GTCACAGAACCCACTCCCGT & This work \\
\hline ASU2-comE1-R1 & & & TTTATCTTGGATTTCCGCTGCTGTT & This work \\
\hline
\end{tabular}


Real-time PCR was done in a LightCycler 480 (Roche Diagnostics Co., Indianapolis, IN) using a program with an initial denaturation of $95^{\circ} \mathrm{C}$ for 5 min followed by 45 cycles of $95{ }^{\circ} \mathrm{C}$ for $10 \mathrm{~s}, 54{ }^{\circ} \mathrm{C}$ for $20 \mathrm{~s}$, and $72{ }^{\circ} \mathrm{C}$ for $12 \mathrm{~s}$. Stepwise melt curves were done at the end of each run to confirm that only one template was amplified.

\section{Sequencing additional isolates}

A. suis strains ATCC 15557, H89-1173, and H91-0406 were sequenced at the Advanced Analytics Centre at the University of Guelph using MiSeq, and pseudogenomes were assembled with SeqMan Pro (DNASTAR Inc., Madison, WI, USA) followed by progressiveMauve [60], and annotated using the BASys pipeline [58].

\section{Additional file}

Additional file 1. blastn comparison of A. suis H91-0380 adhesin-associated genes to four additional $A$. suis strains. Spreadsheet of blastn results showing gene sizes, locations, query coverage, E value, and sequence identity for adhesin-associated genes in A. suis H91-0380 compared to A. suis ATCC 33415, H91-0406, ATCC 15557, and H89-1173.

\section{Authors' contributions}

ARB designed and carried out data collection and analysis, and drafted the manuscript. JIM assisted in experimental design. Both authors read and approved the final manuscript.

\section{Acknowledgements}

The authors thank Glenn Soltes for assistance with Real-time PCR experiments, and Andrew Shure and Dr. Andrew Kropinski for providing pseudogenomes. This work was funded by a grant from the Natural Sciences and Engineering Research Council of Canada to JIM. ARB is supported by an Ontario Veterinary College PhD Scholarship and an Ontario Graduate Scholarship.

\section{Competing interests}

The authors declare that they have no competing interests.

Received: 17 July 2015 Accepted: 2 November 2015

Published online: 14 November 2015

\section{References}

1. Kernaghan S, Bujold AR, Maclnnes Jl. The microbiome of the soft palate of swine. Anim Health Res Rev. 2012;13:110-20.

2. Maclnnes J, Desrosiers R. Agents of the "suis-ide diseases" of swine: actinobacillus suis, Haemophilus parasuis, and Streptococcus suis. Can J Vet Res. 1999;63:83-9.

3. Bosse JT, Janson H, Sheehan BJ, Beddek AJ, Rycroft AN, Kroll JS, Langford PR. Actinobacillus pleuropneumoniae: pathobiology and pathogenesis of infection. Microb Infect. 2002;4:225-35.

4. MacInnes Jl. Actinobacillus. In: Gyles CL, Prescott JF, Songer G, Thoen CO, editors. Pathogenesis of bacterial infections in animals. 4th ed. Ames: Wiley-Blackwell; 2010. p. 363-86.

5. Jeannotte ME, Slavic D, Frey J, Kuhnert P, Maclnnes Jl. Analysis of nonporcine isolates of Actinobacillus suis. Vet Microbiol. 2002;85:83-93.

6. Maclnnes Jl, Mackinnon J, Bujold AR, Ziebell K, Kropinski AM, Nash JH. Complete genome sequence of Actinobacillus suis H91-0380, a virulent serotype O2 strain. J Bacteriol. 2012;194:6686-7.

7. Ofek I, Doyle RJ. Bacterial adhesion to cells and tissues. New York: Chapman \& Hall; 1994.
8. Soto GE, Hultgren SJ. Bacterial adhesins: common themes and variations in architecture and assembly. J Bacteriol. 1999;181:1059-71.

9. Klemm P, Schembri MA. Bacterial adhesins: function and structure. Int J Med Microbiol. 2000;290:27-35.

10. Linke D, Goldman A. Bacterial adhesion: chemistry, biology and physics. Dordrecht: Springer; 2011.

11. Cotter SE, Surana NK, St Geme JW 3rd. Trimeric autotransporters: a distinct subfamily of autotransporter proteins. Trends Microbiol. 2005;13:199-205.

12. Surana NK, Cutter D, Barenkamp SJ, St Geme JW 3rd. The Haemophilus influenzae Hia autotransporter contains an unusually short trimeric translocator domain. J Biol Chem. 2004;279:14679-85.

13. Hoiczyk E, Roggenkamp A, Reichenbecher M, Lupas A, Heesemann J. Structure and sequence analysis of Yersinia YadA and Moraxella UspAs reveal a novel class of adhesins. EMBO J. 2000;19:5989-99.

14. Roggenkamp A, Ackermann N, Jacobi CA, Truelzsch K, Hoffmann H, Heesemann J. Molecular analysis of transport and oligomerization of the Yersinia enterocolitica adhesin YadA. J Bacteriol. 2003;185:3735-44.

15. Watson AA, Alm RA, Mattick JS. Identification of a gene, pilF, required for type 4 fimbrial biogenesis and twitching motility in Pseudomonas aeruginosa. Gene. 1996;180:49-56.

16. Carbonnelle E, Helaine S, Prouvensier L, Nassif X, Pelicic V. Type IV pilus biogenesis in Neisseria meningitidis: PilW is involved in a step occurring after pilus assembly, essential for fibre stability and function. Mol Microbiol. 2005;55:54-64.

17. Cerveny L, Straskova A, Dankova V, Hartlova A, Ceckova M, Staud F, Stulik J. Tetratricopeptide repeat motifs in the world of bacterial pathogens: role in virulence mechanisms. Infect Immun. 2013;81:629-35.

18. Planet PJ, Kachlany SC, Fine DH, DeSalle R, Figurski DH. The widespread colonization island of Actinobacillus actinomycetemcomitans. Nat Genet. 2003;34:193-8.

19. Spinola SM, Fortney KR, Katz BP, Latimer JL, Mock JR, Vakevainen M, Hansen EJ. Haemophilus ducreyi requires an intact flp gene cluster for virulence in humans. Infect Immun. 2003;71:7178-82.

20. Fuller TE, Kennedy MJ, Lowery DE. Identification of Pasteurella multocida virulence genes in a septicemic mouse model using signature-tagged mutagenesis. Microb Pathog. 2000;29:25-38.

21. Tomich M, Fine DH, Figurski DH. The TadV protein of Actinobacillus actinomycetemcomitans is a novel aspartic acid prepilin peptidase required for maturation of the Flp1 pilin and TadE and TadF pseudopilins. J Bacteriol. 2006;188:6899-914

22. Tomich M, Planet PJ, Figurski DH. The tad locus: postcards from the widespread colonization island. Nat Rev Microbiol. 2007;5:363-75.

23. LiT, Xu Z, Zhang T, Li L, Chen H, Zhou R. The genetic analysis of the flp locus of Actinobacillus pleuropneumoniae. Arch Microbiol. 2012;194:167-76.

24. Carruthers MD, Tracy EN, Dickson AC, Ganser KB, Munson RS Jr, Bakaletz LO. Biological roles of nontypeable Haemophilus influenzae type IV pilus proteins encoded by the pil and com operons. J Bacteriol. 2012;194:1927-33.

25. Bakaletz LO, Baker BD, Jurcisek JA, Harrison A, Novotny LA, Bookwalter JE, Mungur R, Munson RS Jr. Demonstration of Type IV pilus expression and a twitching phenotype by Haemophilus influenzae. Infect Immun. 2005;73:1635-43.

26. Jurcisek JA, Bakaletz LO. Biofilms formed by nontypeable Haemophilus influenzae in vivo contain both double-stranded DNA and type IV pilin protein. J Bacteriol. 2007;189:3868-75.

27. Jurcisek JA, Bookwalter JE, Baker BD, Fernandez S, Novotny LA, Munson RS Jr, Bakaletz LO. The PilA protein of non-typeable Haemophilus influenzae plays a role in biofilm formation, adherence to epithelial cells and colonization of the mammalian upper respiratory tract. Mol Microbiol. 2007;65:1288-99.

28. Hunt ML, Adler B, Townsend KM. The molecular biology of Pasteurella multocida. Vet Microbiol. 2000;72:3-25.

29. Boekema BK, Stockhofe-Zurwieden N, Smith HE, Kamp EM, van Putten $J \mathrm{P}$, Verheijden JH. Adherence of Actinobacillus pleuropneumoniae to primary cultures of porcine lung epithelial cells. Vet Microbiol. 2003;93:133-44.

30. Boekema BK, Van Putten JP, Stockhofe-Zurwieden N, Smith HE. Host cell contact-induced transcription of the type IV fimbria gene cluster of Actinobacillus pleuropneumoniae. Infect Immun. 2004;72:691-700. 
31. Zhou Y, Li L, Chen Z, Yuan H, Chen H, Zhou R. Adhesion protein ApfA of Actinobacillus pleuropneumoniae is required for pathogenesis and is a potential target for vaccine development. Clin Vaccine Immunol. 2013:20:287-94

32. Wall D, Kaiser D. Type IV pili and cell motility. Mol Microbiol. 1999;32:1-10.

33. Craig L, Pique ME, Tainer JA. Type IV pilus structure and bacterial pathogenicity. Nat Rev Microbiol. 2004;2:363-78.

34. Hansen JK, Forest KT. Type IV pilin structures: insights on shared architecture, fiber assembly, receptor binding and type II secretion. J Mol Microbiol Biotechnol. 2006;11:192-207.

35. Galdiero S, Falanga A, Cantisani M, Tarallo R, Della Pepa ME, D'Oriano V, Galdiero M. Microbe-host interactions: Structure and role of Gram-negative bacterial porins. Curr Protein Peptide Sci. 2012;13:843-54.

36. Ojha S, Lacouture S, Gottschalk M, Maclnnes Jl. Characterization of colonization-deficient mutants of Actinobacillus suis. Vet Microbiol. 2010;140:122-30.

37. Ojha S, Sirois M, Macinnes Jl. Identification of Actinobacillus suis genes essential for the colonization of the upper respiratory tract of swine. Infect Immun. 2005;73:7032-9.

38. Novotny LA, Jurcisek JA, Pichichero ME, Bakaletz LO. Epitope mapping of the outer membrane protein P5-homologous fimbrin adhesin of nontypeable Haemophilus influenzae. Infect Immun. 2000;68:2119-28.

39. Hill DJ, Toleman MA, Evans DJ, Villullas S, Van Alphen L, Virji M. The variable P5 proteins of typeable and non-typeable Haemophilus influenzae target human CEACAM1. Mol Microbiol. 2001;39:850-62.

40. Regelink AG, Dahan D, Moller LV, Coulton JW, Eijk P, Van Ulsen P, Dankert J, Van Alphen L. Variation in the composition and pore function of major outer membrane pore protein P2 of Haemophilus influenzae from cystic fibrosis patients. Antimicrob Agents Chemother. 1999;43:226-32.

41. Andersen C, Maier E, Kemmer G, Blass J, Hilpert AK, Benz R, Reidl J. Porin OmpP2 of Haemophilus influenzae shows specificity for nicotinamidederived nucleotide substrates. J Biol Chem. 2003;278:24269-76.

42. Reddy MS, Bernstein JM, Murphy TF, Faden HS. Binding between outer membrane proteins of nontypeable Haemophilus influenzae and human nasopharyngeal mucin. Infect Immun. 1996;64:1477-9.

43. Locht C, Antoine R, Jacob-Dubuisson F. Bordetella pertussis, molecular pathogenesis under multiple aspects. Curr Opin Microbiol. 2001;4:82-9.

44. Nicholson TL, Brockmeier SL, Loving CL. Contribution of Bordetella bronchiseptica filamentous hemagglutinin and pertactin to respiratory disease in swine. Infect Immun. 2009;77:2136-46.

45. Sandal I, Shao JQ, Annadata S, Apicella MA, Boye M, Jensen TK, Saunders GK, Inzana TJ. Histophilus somni biofilm formation in cardiopulmonary tissue of the bovine host following respiratory challenge. Microbes Infect. 2009;11:254-63.

46. Darvish Alipour Astaneh S, Rasooli I, Mousavi Gargari SL. The role of filamentous hemagglutinin adhesin in adherence and biofilm formation in Acinetobacter baumannii ATCC19606. Microb Pathog. 2014;74C:42-9.

47. Verma S, Sharma M, Katoch S, Verma L, Kumar S, Dogra V, Chahota R, Dhar $P$, Singh G. Profiling of virulence associated genes of Pasteurella multocida isolated from cattle. Vet Res Commun. 2013;37:83-9.

48. Brentjens RJ, Ketterer M, Apicella MA, Spinola SM. Fine tangled pili expressed by Haemophilus ducreyi are a novel class of pili. J Bacteriol. 1996;178:808-16.

49. Haikarainen T, Papageorgiou AC. Dps-like proteins: structural and functional insights into a versatile protein family. Cell Mol Life Sci. 2010;67:341-51.
50. Huergo LF, Rahman H, Ibrahimovic A, Day CJ, Korolik V. Campylobacter jejuni Dps protein binds DNA in the presence of iron or hydrogen perox ide. J Bacteriol. 2013;195:1970-8.

51. Theoret JR, Cooper KK, Glock RD, Joens LA. A Campylobacter jejuni Dps homolog has a role in intracellular survival and in the development of campylobacterosis in neonate piglets. Foodborne Pathog Dis. 2011;8:1263-8.

52. Theoret JR, Cooper KK, Zekarias B, Roland KL, Law BF, Curtiss R 3rd, Joens $L A$. The Campylobacter jejuni Dps homologue is important for in vitro biofilm formation and cecal colonization of poultry and may serve as a protective antigen for vaccination. Clin Vaccine Immunol. 2012;19:1426-31.

53. Pang B, Hong W, Kock ND, Swords WE. Dps promotes survival of nontypeable Haemophilus influenzae in biofilm communities in vitro and resistance to clearance in vivo. Front Cell Infect Microbiol. 2012;2:58.

54. Mullen LM, Nair SP, Ward JM, Rycroft AN, Williams RJ, Henderson B. Comparative functional genomic analysis of Pasteurellaceae adhesins using phage display. Vet Microbiol. 2007;122:123-34.

55. Mullen LM, Bosse JT, Nair SP, Ward JM, Rycroft AN, Robertson G, Langford PR, Henderson B. Pasteurellaceae ComE1 proteins combine the properties of fibronectin adhesins and DNA binding competence proteins. PLoS One. 2008;3:e3991.

56. Mullen LM, Nair SP, Ward JM, Rycroft AN, Williams RJ, Robertson G, Mordan NJ, Henderson B. Novel adhesin from Pasteurella multocida that binds to the integrin-binding fibronectin FnIII9-10 repeats. Infect Immun. 2008;76:1093-104.

57. Calcutt MJ, Foecking MF, Mhlanga-Mutangadura T, Reilly TJ. Genome sequence of Actinobacillus suis type strain ATCC $33415^{\top}$. Genome Announc 2014; 2

58. Van Domselaar GH, Stothard P, Shrivastava S, Cruz JA, Guo A, Dong X, Lu P, Szafron D, Greiner R, Wishart DS. BASys: a web server for automated bacterial genome annotation. Nucleic Acids Res. 2005;33:W455-9.

59. Thornton $B$, Basu C. Real-time PCR (qPCR) primer design using free online software. Biochem Mol Biol Educ. 2011;39:145-54.

60. Darling AE, Mau B, Perna NT. progressiveMauve: multiple genome alignment with gene gain, loss and rearrangement. PLoS One. 2010;5:e11147.

61. Van Ostaaijen J, Frey J, Rosendal S, Maclnnes Jl. Actinobacillus suis strains isolated from healthy and diseased swine are clonal and carry apxICABDvar. suis and apxI/CA $A_{\text {var. suis }}$ toxin genes. J Clin Microbiol. 1997;35:1131-7.

62. Slavic D, DeLay J, Hayes MA, Maclnnes Jl. Comparative pathogenicity of different Actinobacillus suis O/K serotypes. Can J Vet Res. 2000;64:81-7.

63. Foote SJ, Bosse JT, Bouevitch AB, Langford PR, Young NM, Nash JH. The complete genome sequence of Actinobacillus pleuropneumoniae L20 (serotype 5b). J Bacteriol. 2008;190:1495-6.

\section{Submit your next manuscript to BioMed Central and take full advantage of:}

- Convenient online submission

- Thorough peer review

- No space constraints or color figure charges

- Immediate publication on acceptance

- Inclusion in PubMed, CAS, Scopus and Google Scholar

- Research which is freely available for redistribution 